\title{
Correlative Imaging of Hypoxia and Angiogenesis in Oncology
}

H tumor development and aggressiveness and is an important prognostic factor for resistance to antineoplastic treatments. Hypoxic cells in tumors are about 3-fold more resistant to radiation therapy than their well-oxygenated counterparts (1). In the early stage of development, tumors remain dormant in an avascular phase, with active cellular proliferation balanced by cell apoptosis. The phenomenon, in which a tumor progresses from a nonangiogenic to angiogenic phenotype, has been termed the angiogenic switch (2). Hypoxia is a key player in the angiogenic

\section{See page 597}

switch during tumor development. A key regulator of hypoxia-induced angiogenesis is the hypoxia-inducible factor 1 (HIF-1). Multiple HIF-1 target genes have been shown to modulate angiogenesis by promoting the mitogenic and migratory activities of endothelial cells (3). Accumulating evidence, however, indicates that HIF-1-independent pathways can also control angiogenesis (4). Hypoxiainduced angiogenesis has become an important area in basic, translational, and clinical cancer research and an attractive target for cancer therapy (5). The mechanisms involved during this process and how best to target it for

Received Feb. 6, 2008; revision accepted Feb. 20, 2008.

For correspondence contact: Karl-Josef Langen, $\mathrm{MD}$, Institute of Neuroscience and BiophysicsMedicine, Research Center Jülich, D-52425 Jülich, Germany.

E-mail: k.j.langen@fz-juelich.de

COPYRIGHT (C) 2008 by the Society of Nuclear

Medicine, Inc.

DOI: 10.2967/jnumed.108.050559 cancer therapy are still under investigation. Oxygenation therapy, hypoxiadirected intensity-modulated radiotherapy, and antiangiogenic therapy in combination with cytotoxic chemotherapy are some of the current approaches to improve the therapeutic success in various tumor entities $(2,5,6)$. Consequently, there is a growing demand for imaging modalities that allow for pretherapeutic stratification and response assessment of patients receiving targeted therapies.

In this issue of The Journal of Nuclear Medicine, Picchio et al. (7) report on the spatial distribution of hypoxia and angiogenesis in rat tumor xenografts using double-tracer autoradiography. The authors applied the second-generation nitroimidazole-based hypoxia tracer ${ }^{18} \mathrm{~F}$ fluoroazomycin arabinoside (FAZA) and ${ }^{125}$ I-gluco-RGD, which binds with high affinity to the $\alpha_{\mathrm{v}} \beta_{3}$-integrin receptors that are highly expressed on activated endothelial cells during angiogenesis. A corresponding PET tracer, that is, ${ }^{18} \mathrm{~F}$ galacto-RGD, is currently under clinical evaluation $(8,9)$.

The comparison of ${ }^{18} \mathrm{~F}$-FAZA distribution with immunohistochemical staining for HIF-1 as a gold standard of tissue hypoxia demonstrated that HIF-1 staining underestimated the extent of hypoxia as determined by ${ }^{18} \mathrm{~F}$ FAZA. The authors put the role of HIF-1 as a surrogate marker for hypoxia into question, as is supported by several other publications (7). Roughly $60 \%$ of the tumor surface displayed the expected coupling between hypoxia and angiogenesis, that is, both low ${ }^{18} \mathrm{~F}$ FAZA and ${ }^{125}$ I-gluco-RGD uptake on the one hand or elevated ${ }^{18} \mathrm{~F}$-FAZA and ${ }^{125}$ I-gluco-RGD uptake on the other hand. However, approximately $40 \%$ of the tumor surface showed a discordant pattern indicating that hypoxia and angiogenesis are not necessarily spatially linked in malignant tumors. The authors speculate that tumor areas with increased ${ }^{18} \mathrm{~F}$-FAZA uptake and low ${ }^{125}$ I-gluco-RGD uptake may indicate acute hypoxia when angiogenesis is not yet activated. In contrast, areas with low ${ }^{18}$ F-FAZA uptake and high ${ }^{125}$ I-glucoRGD uptake may indicate tumor areas that have escaped hypoxia because of activated angiogenesis.

Certainly, this concept needs further confirmation, especially given the limited specificity of $\alpha_{\mathrm{v}} \beta_{3}$-integrin ligands for imaging of tumor angiogenesis and the fact that the results concerning angiogenesis were not confirmed by a standard immunohistochemical method. Nevertheless, the study by Picchio et al. highlights the possible contribution of combined in vivo imaging of hypoxia and angiogenesis before and during therapy to the optimization of targeted chemotherapeutic and antiangiogenic therapy approaches in individual patients. With the rapid development and spread of integrated PET and CT scanners featuring a capability for full planning of radiation therapy, and specific PET tracers that are able to target hypoxia and angiogenesis in vivo, the required tools to integrate these parameters into clinical research and practice are becoming more and more available.

Although these experimental results are rather encouraging, a short consideration of the tracers that are currently under clinical evaluation for in vivo imaging of hypoxia and angiogenesis with PET is needed. At present, most experience concerning noninvasive imaging of tumor hypoxia is available for ${ }^{18} \mathrm{~F}$-labeled fluoromisonidazole (10). This tracer has successfully been applied in numerous studies and turned 
out to be useful to predict the outcome of radiotherapy in lung and head and neck cancers and to detect tumor reoxygenation during radiotherapy (10-12). Recently, the evaluation of ${ }^{18} \mathrm{~F}$-fluoromisonidazole kinetics was found to be helpful to characterize the hypoxia-perfusion pattern of head and neck tumors - a pattern that correlated with the outcome of radiotherapy $(12,13)$. Nevertheless, the tracer is not optimal for clinical application because of slow accumulation in hypoxic tumors, a low tumor-to-background (T/B) contrast, and a significant amount of radioactive metabolites (14). The secondgeneration hypoxia tracer ${ }^{18} \mathrm{~F}-\mathrm{FAZA}$ exhibits a faster clearance than ${ }^{18} \mathrm{~F}$ fluoromisonidazole and higher T/B contrast in animal experiments $(15,16)$. A first study on patients with head and neck cancer, however, showed only slightly increased $\mathrm{T} / \mathrm{B}$ ratios at $2 \mathrm{~h}$ after injection, compared with ${ }^{18} \mathrm{~F}-\mathrm{MISO}$ $(14,17)$. In many tumors, the $\mathrm{T} / \mathrm{B}$ ratios of ${ }^{18}$ F-FAZA uptake decreased between 2 and $4 \mathrm{~h}$ after injection, indicating that ${ }^{18} \mathrm{~F}-\mathrm{FAZA}$ binding to hypoxic tumor tissue is not irreversible. Although ${ }^{18} \mathrm{~F}$ FAZA shows clinical potential similar to that of ${ }^{18}$ F-fluoromisonidazole $(18,19)$, there are only minor advantages, and further efforts for the development of an optimal hypoxia tracer with higher T/B contrast are needed.

The field of angiogenesis is a rapidly growing biomedical discipline, and great efforts are being undertaken to develop antiangiogenesis drugs for the treatment of cancer. One target structure is the $\alpha_{v} \beta_{3}$ integrin receptor, which is highly expressed on activated endothelial cells during angiogenesis. Various ligands based on the tripeptide RGD, which binds with high affinity to the $\alpha_{v} \beta_{3}$-integrin receptor, have been developed for SPECT and PET (20). The glycosylated cyclic pentapeptide ${ }^{18} \mathrm{~F}$-galacto-RGD resulted from tracer optimization based on the first-generation peptide ${ }^{125}$ I-3-iodo-d-Tyr4-cyclo(-ArgGly-Asp-d-Tyr-Val-) (21). Initial clinical studies using ${ }^{18} \mathrm{~F}$-galacto-RGD and PET demonstrated that tracer accumulation in the tumor correlates with $\alpha_{v} \beta_{3}$ expression but also revealed high inter- and intraindividual variance in tracer accumulation in tumors, indicating great diversity in receptor expression $(8,9)$. In a comparative study, no correlation between ${ }^{18} \mathrm{~F}-\mathrm{FDG}$ and ${ }^{18} \mathrm{~F}$ galacto-RGD tracer uptake in the tumors was found, and the sensitivity of ${ }^{18} \mathrm{~F}$-galacto-RGD for tumor detection was clearly inferior to that of ${ }^{18} \mathrm{~F}-\mathrm{FDG}$ (9). Another limitation is that $\alpha_{\mathrm{v}} \beta_{3}$ can be expressed on tumor cells as well as on endothelial cells, making it difficult to attribute the resulting signal exclusively to molecular processes during tumor-induced angiogenesis. Furthermore, the exact role of $\alpha_{\mathrm{v}} \beta_{3}$ expression in the context of angiogenesis is still a matter of discussion. Experiments on knockout mice lacking the integrin $\alpha_{v} \beta_{3}$ led to a reevaluation of the role of $\alpha_{\mathrm{v}} \beta_{3}$ with regard to angiogenesis, because the knockout mice showed normal developmental angiogenesis and even excessive tumor angiogenesis (22).

In summary, molecular imaging of hypoxia and angiogenesis using PET is an exciting field that has made considerable progress in recent years and may soon contribute significantly to individually targeted therapies. The tracers currently available already have a high potential to approach these goals, but further efforts in the development of new ligands with higher T/B contrast and specificity are desirable.

Karl Josef Langen
Research Center Jülich
Jülich, Germany

\section{Susanne Martina Eschmann Marienhospital Stuttgart Stuttgart Germany}

\section{REFERENCES}

1. Horsman MR, Overgaard J. The oxygen effect and tumour microenvironment. In: Steel GG, ed. Basic Clinical Radiobiology. London, U.K.: Arnold; 2002:158-168.

2. Liao D, Johnson RS. Hypoxia: a key regulator of angiogenesis in cancer. Cancer Metastasis Rev. 2007;26:281-290.

3. Pugh CW, Ratcliffe PJ. Regulation of angiogenesis by hypoxia: role of the HIF system. Nat Med. 2003;9:677-684.

4. Mizukami Y, Kohgo Y, Chung DC. Hypoxia inducible factor-1 independent pathways in tumor angiogenesis. Clin Cancer Res. 2007;13:5670 5674.
5. Diaz-Gonzalez JA, Russell J, Rouzaut A, Gil-Bazo I, Montuenga L. Targeting hypoxia and angiogenesis through HIF-1alpha inhibition. Cancer Biol Ther. 2005;4:1055-1062.

6. Lyseng-Williamson KA, Robinson DM. Spotlight on bevacizumab in advanced colorectal cancer, breast cancer, and non-small cell lung cancer. BioDrugs. 2006;20:193-195.

7. Picchio M, Beck R, Haubner R, et al. Intratumoral spatial distribution of hypoxia and angiogenesis assessed by ${ }^{18} \mathrm{~F}$-FAZA and ${ }^{125} \mathrm{I}$-gluco-RGD autoradiography. J Nucl Med. 2008;49:597-605.

8. Beer AJ, Haubner R, Goebel M, et al. Biodistribution and pharmacokinetics of the alpha beta $_{3}$ selective tracer ${ }^{18} \mathrm{~F}$-galacto-RGD in cancer patients. J Nucl Med. 2005;46:1333-1341.

9. Beer AJ, Lorenzen S, Metz S, et al. Comparison of integrin $\alpha_{v} \beta_{3}$-expression and glucose metabolism in primary and metastatic lesions in cancer patients: A PET study using ${ }^{18} \mathrm{~F}$-galacto-RGD and ${ }^{18}$ F-FDG. J Nucl Med. 2008;49:22-29.

10. Lee ST, Scott AM. Hypoxia positron emission tomography imaging with ${ }^{18} \mathrm{~F}$-fluoromisonidazole. Semin Nucl Med. 2007;37:451-461.

11. Eschmann SM, Paulsen F, Reimold M, et al. Prognostic impact of hypoxia imaging with ${ }^{18} \mathrm{~F}$ misonidazole PET in non-small cell lung cancer and head and neck cancer before radiotherapy. J Nucl Med. 2005;46:253-260.

12. Eschmann SM, Paulsen F, Bedeshem C, et al. Hypoxia-imaging with ${ }^{18} \mathrm{~F}$-misonidazole and PET: changes of kinetics during radiotherapy of headand-neck cancer. Radiother Oncol. 2007;83:406-410.

13. Thorwarth D, Eschmann SM, Scheiderbauer J, Paulsen F, Alber M. Kinetic analysis of dynamic ${ }^{18}$ F-fluoromisonidazole PET correlates with radiation treatment outcome in head-and-neck cancer. BMC Cancer. 2005;5:152.

14. Grönroos T, Minn H. Imaging of tumour hypoxia using PET and ${ }^{18}$ F-labelled tracers: biology meets technology. Eur J Nucl Med Mol Imaging. 2007; 34:1563-1565.

15. Sorger D, Patt M, Kumar P, et al. $\left[{ }^{18} \mathrm{~F}\right]$ Fluoroazomycinarabinofuranoside $\left({ }^{18} \mathrm{FAZA}\right)$ and $\left[{ }^{18} \mathrm{~F}\right]$ fluoromisonidazole ( $\left.{ }^{18} \mathrm{FMISO}\right)$ : a comparative study of their selective uptake in hypoxic cells and PET imaging in experimental rat tumors. Nucl Med Biol. 2003;30:317-326.

16. Piert M, Machulla HJ, Picchio M, et al. Hypoxiaspecific tumor imaging with ${ }^{18} \mathrm{~F}$-fluoroazomycin arabinoside. J Nucl Med. 2005;46:106-113.

17. Souvatzoglou M, Grosu AL, Röper B, et al. Tumour hypoxia imaging with $\left[{ }^{18} \mathrm{~F}\right] \mathrm{FAZA}$ PET in head and neck cancer patients: a pilot study. Eur J Nucl Med Mol Imaging. 2007;34:1566-1575.

18. Grosu AL, Souvatzoglou M, Röper B, et al. Hypoxia imaging with FAZA-PET and theoretical considerations with regard to dose painting for individualization of radiotherapy in patients with head and neck cancer. Int $J$ Radiat Oncol Biol Phys. 2007 1;69:541-551

19. Beck R, Röper B, Carlsen JM, et al. Pretreatment ${ }^{18}$ F-FAZA PET predicts success of hypoxiadirected radiochemotherapy using tirapazamine. J Nucl Med. 2007;48:973-980.

20. Haubner R. Alpha beta $_{3}$-integrin imaging: a new approach to characterise angiogenesis? Eur J Nucl Med Mol Imaging. 2006;33(suppl 1):54-63.

21. Haubner R, Wester HJ, Reuning U, et al. Radiolabeled alpha $_{v}$ beta $_{3}$ integrin antagonists: a new class of tracers for tumor targeting. J Nucl Med. 1999;40:1061-1071.

22. Hynes RO. A reevaluation of integrins as regulators of angiogenesis. Nat Med. 2002;8:918-921. 